\title{
The Effect of Spironolactone on the Incidence of Contrast-Induced Nephropathy in Patients Undergoing Cardiac Catheterization: Study Design and Rationale
}

\author{
Alhasan Mujtaba (D) - Mohammed A. Taher - Mazin A. Hazza • \\ Hassan M. Al-Rubaye - Asaad H. Kata - Hamid AbdulWahab • \\ AbdulAmeer AbdulBari · Hayder K. AlRubay
}

Received: April 11, 2018 / Published online: May 21, 2018

(C) The Author(s) 2018

\begin{abstract}
Introduction: Patients undergoing coronary catheterization are at high risk of developing contrast-induced nephropathy (CIN) acute kidney injury (AKI). Several approaches have been supposed to limit such an effect but with mixed results or non-practical methods. Spironolactone is supposed to be effective as a nephroprotective agent in animal studies. This study will try to measure the effect of spironolactone on the incidence of CIN-AKI in patients undergoing coronary catheterization (angiography angioplasty).
\end{abstract}

Methods: This study is a single-center, investigator-driven, double-blinded randomized controlled study in Iraq-Basra. More than 400 patients admitted for coronary angio unit in our

Enhanced digital features To view enhanced digital features for this article go to https://doi.org/10.6084/ m9.figshare.6244637.

A. Mujtaba $(\bowtie) \cdot$ M. A. Taher

Department of Clinical Pharmacy, College of

Pharmacy, University of Baghdad, Basra, Iraq

e-mail: user11126@gmail.com

M. A. Hazza · A. AbdulBari

College of Medicine, University of Basra, Basra, Iraq

H. M. Al-Rubaye · A. H. Kata - H. AbdulWahab Basra Cardiac Catheterization Center, Basra, Iraq

H. K. AlRubay

AlSader Teaching Hospital, Basra, Iraq center will be allocated in a 1:1 ratio to receive either spironolactone $200 \mathrm{mg}$ single dose or placebo in addition to their usual premedication.

Planned Outcomes: Primary end point will be CIN defined as more than $25 \%$ or $0.3 \mathrm{mg} / \mathrm{dl}$ elevation in serum creatinine (S.Cr.) from baseline during the first 2-3 days after the procedure. We hope to identify or answer an important question regarding CIN in such highrisk patients.

Trial Registration: ClinicalTrials.gov Identifier, NCT03329443.

Keywords: Acute kidney injury (AKI); Aldactone; Angiography; Angioplasty; Contrast-induced nephropathy (CIN); Ischemic heart disease (IHD); Percutaneous coronary angiography (PCI); Spironolactone

\section{INTRODUCTION}

Patients who opt for percutaneous coronary intervention (PCI) to help them with their ischemic heart disease (IHD) problems are at high risk of developing contrast-induced nephropathy (CIN) [1]. The CIN could ultimately have a detrimental effect on such patients including a negative effect on mortality and morbidity as well $[2,3]$.

Several interventions have been done to limit this negative effect on such patients, but 
the evidence is still lacking on the best method, and the maximum benefit that can be achieved to prevent CIN. Several approaches may include aggressive hydration prior to the procedure, but results are still pending [4-6]. Furthermore, it has been reported that another innovative approach was based on blocking the neurohormonal activation known to cause or aggravate acute kidney injury (AKI). One such approach is the use of spironolactone, where animal studies highlighted the damaging effect of aldosterone on causing and aggravating AKI and specifically CIN. On the other hand, human studies are conflicting and importantly no study yet have been done on patients with CIN or PCI [7-10].

Given all the previous information, we designed this study to measure the effect of aldosterone blockage promoted by the utilization of spironolactone prior to coronary angiography on CIN incidence measured by different biochemical approaches and definitions that were reported in the literature and adopted by global societies.

\section{METHODS}

\section{Study Population}

This study (trial registration: ClinicalTrials.gov NCT03329443) will be an investigator-initiated, single-center, double-blind placebo-controlled, randomized clinical trial. Trial design was approved by two independent institutional review boards (Basra Health directorate review board, and University of Baghdad/College of Pharmacy review board) before patients' enrollment in the study. Patients admitted for coronary angio in the cardiology department in Al-Sader teaching hospital-Basra will be selected for randomization by a computer-based algorithm into two groups. The study population will consist of $400+$ patients 18 years or older, admitted for coronary angiography or PCI and provide informed consent for participation in the study. Patients will be excluded if they received contrast medium administration within the previous 7 days, had end-stage renal failure or kidney transplantation, acute kidney injury defined as $0.3 \mathrm{mg} / \mathrm{dl}$ elevation in serum creatinine (S.Cr.) in the previous 4 weeks, lactation, pregnancy or documented current tumor, periprocedural administration of nephrotoxic drugs, (nonsteroidal anti-inflammatory drugs, cyclosporine, aminoglycosides or cisplatin in the previous 48 days or in the follow-up period).

\section{Study Protocol}

We are using an Excel spreadsheet with random number generator to allocate eligible patients in a 1:1 ratio for either active or placebo groups. Simple randomization is selected without any stratification, which was felt to be adequate.

The active arm allocated group will receive $200 \mathrm{mg}$ of spironolactone prior to angiography/plasty at admission along with their premedication and chronic treatments. The placebo group will receive only their usual premedication and chronic treatment.

Blood samples will be collected for assessment of serum creatinine, NGAL (neutrophil gelatinase-associated lipocalin), potassium, and hematocrit at baseline. Serum NGAL and potassium is further collected after $6 \mathrm{~h}$ of the procedure. Finally, patients are advised to report serum creatinine after $48-72 \mathrm{~h}$ post procedure. Patients who fail to report will receive a reminder text message within $48-72 \mathrm{~h}$ to enhance feedback (Fig. 1).

Patients' demographic data, including phone number, weight, age, gender, type, and volume of contrast agent received in the procedure, type of procedure (PCI/angio), lesion location, hypertension, DM, chronic kidney disease stage by Cockcroft-Gault equation, Mehran score, type and fluid given in the peri-procedure, current medication including spironolactone and any nephrotoxic drugs like metformin and diuretics will be carefully collected by the research personnel.

\section{Compliance with Ethics Guidelines}

All procedures performed in this study involving human participants will be in accordance with the ethical standards of the institutional 


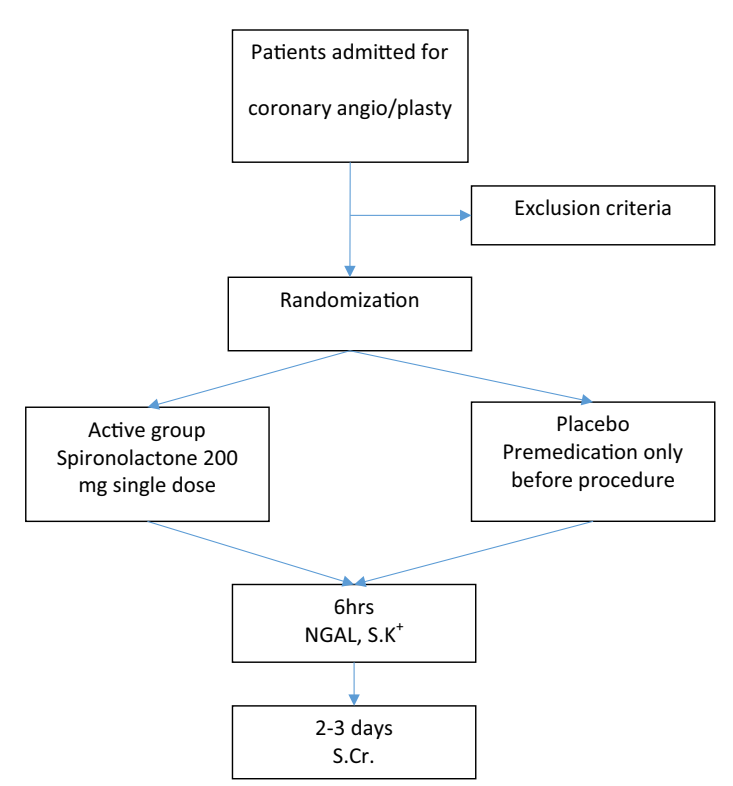

Fig. 1 Study flow diagram. $N G A L$ neutrophil gelatinaseassociated lipocalin, S.K $K^{+}$serum potassium, S.Cr. serum creatinine

and/or national research committee and with the 1964 Helsinki Declaration and its later amendments or comparable ethical standards. Informed consent will be obtained from all individual participants included in the study.

\section{Study Status}

We are ongoing now with an estimated enrollment rate of more than 20 patients per week from five consultant cardiologists out of seven in our center.

\section{Study End Points}

\section{Primary Endpoint}

The primary outcome will be a documentation of CIN defined as $0.25 \%$ or $0.3 \mathrm{mg}$ absolute increase in Sr. Cr. after $48-72 \mathrm{~h}$ after the procedure as adopted by the K-DIGO guidelines $[11,12]$.

\section{Key Secondary Endpoints}

Key secondary endpoints will be an evaluation of CIN by the novel surrogate marker serum NGAL after only $6 \mathrm{~h}$ post procedure [13].
As a safety end point and because of inherent risks of hyperkalemia, patients will be followed up for hyperkalemia for $6 \mathrm{~h}$ after procedure and contacted in 2-3 days to look for any signs and symptoms of hyperkalemia and they will be specifically advised to immediately measure serum potassium level if any signs hyperkalemia would develop or a documented gastrointestinal tract (GIT) upset in the same period $[14,15]$.

\section{Data and Safety Monitoring Board}

Data will be sent periodically to the principal administrators of the study to look for efficacy, safety, and the will to continue or terminate the study.

\section{Statistical Analysis}

Sample size calculation for our study was based on our pilot observation (unpublished yet) and other studies reported in similar Iraqi patients from nearby centers with a CIN incidence in the control group of around $20 \%$ and an assumable effect of $10 \%$ reduction with spironolactone $[16,17]$. Using GPower 3.1 with two-sided Chisquare test and an alpha error of 0.05 , a total of 428 patients are required to give at least an $80 \%$ power for our analysis to detect a difference at the specified effect.

Primary and secondary analysis of categorical variables will be conducted using Chi-square test or a proper exact test if necessary. A multivariate logistic regression model will be developed to adjust for a priori specified clinical variables with known influence of CIN as were defined by previous studies [2, 18, 19].

For normally distributed continuous variables, we will adopt a mean + standard deviation (SD) and comparisons will be done with two-sample $t$ tests. Non-normally distributed variables will be presented as median and interquartile range, and analysis will be used according to a suitable non-parametric test if necessary.

All analyses will be done on an intention-totreat principle. All tests will be two-tailed and a $p$ value of $<0.05$ will be considered statistically 
significant. Data analysis will be performed using SPSS 23 software.

\section{SUMMARY}

This study will try to measure the effect of spironolactone as an add-on pre-PCI treatment to limit the effect of CIN in stable IHD patients opting for angiography for diagnosis and treatment.

\section{DISCUSSION}

As mentioned earlier, there are numerous efforts to limit the damaging effects of coronary intervention and specifically the contrast agents on the kidneys [4-6]. Several of such approaches have failed, proved to be non-practical, or gave mixed results at best [20,21]. Spironolactone is poorly studied in this regard given its proven benefit in animal studies [7-9]; two studies in cardiac surgery units reported its effect on ischemic kidney injury. One study was not primarily powered to detect that effect, and the other was underpowered and groups were not homogenous for important covariates relevant to the effect $[7,10]$. There is no study yet so far, registered or published, that studied this drug in coronary angio patients. We aim to measure such an effect in our planned study in a broad category of patients who are admitted for elective coronary angio.

Physicians were given the freedom of treatment regarding prevention of CIN with fluids or any other means but all of these variables will be collected as well. A promising method for CIN prevention might be based on the POSEIDON protocol, but it is difficult to come up with in practical terms and will not be done in our trial due to procedure limitation. Nevertheless, any fluid given will be collected and any other method of CIN prevention will be monitored and collected too [21].

The studies that aimed to measure the effect of spironolactone did so with minimal doses (7, r10). Given the max chronic dose of $400 \mathrm{mg}$, we thought that a higher dose (200-mg single dose) might give a more noticeable effect and still be within the safe range, especially to note that it will be a single dose only.

Nevertheless, we will measure the effect of this moderate dose on serum potassium (S.K+) after $6 \mathrm{~h}$ in all patients and possibly after 3 days in randomly selected patients. Many studies reported the safety of moderate acute (single or few days) dose on serum potassium levels and chronic follow-up of such levels were unnecessary [22-24]. If hyperkalemia were to be found, it will be reported to the treating physician immediately for follow-up and treatment according local guidelines.

\section{ACKNOWLEDGEMENTS}

Funding. No funding or sponsorship was/ will be received for this study or publication of this article. The article processing charges were funded by the authors.

Authorship. All named authors meet the International Committee of Medical Journal Editors (ICMJE) criteria for authorship for this article, take responsibility for the integrity of the work as a whole, and have given their approval for this version to be published.

Disclosures. Alhasan Mujtaba, Mohammed A. Taher, Mazin A. Hazza, Hassan M. Al-Rubaye, Asaad H. Kata, Hamid AbdulWahab, Abdu1Ameer AbdulBari and Hayder K. AlRubay have nothing to disclose. Alhasan Mujtaba, Mohammed A. Taher, Mazin A. Hazza, Hassan M. AlRubaye, Asaad H. Kata, Hamid AbdulWahab, AbdulAmeer AbdulBari and Hayder K. AlRubay alone, are responsible for the writing and production of the present article.

Compliance with Ethics Guidelines. All procedures performed in this study involving human participants will be in accordance with the ethical standards of the institutional and/or national research committee and with the 1964 Helsinki Declaration and its later amendments or comparable ethical standards. Informed consent will be obtained from all individual participants included in the study. 
Data Availability. Data sharing is not applicable to this article as no datasets were generated or analyzed during the current study.

Open Access. This article is distributed under the terms of the Creative Commons Attribution-NonCommercial 4.0 International License (http://creativecommons.org/licenses/ by-nc/4.0/), which permits any noncommercial use, distribution, and reproduction in any medium, provided you give appropriate credit to the original author(s) and the source, provide a link to the Creative Commons license, and indicate if changes were made.

\section{REFERENCES}

1. Tehrani S, et al. Contrast-induced acute kidney injury following PCI. Eur J Clin Invest. 2013;43:483-90.

2. Tziakas D, et al. Validation of a new risk score to predict contrast-induced nephropathy after percutaneous coronary intervention. Am J Cardiol. 2014;113:1487-93.

3. Sadeghi HM, Stone GW, Grines CL, et al. Impact of renal insufficiency in patients undergoing primary angioplasty for acute myocardial infarction. Circulation. 2003;108:2769-75.

4. Liu M, et al. Aggressive hydraTion in patients with ST-elevation myocardial infarction undergoing primary percutaneous coronary intervention to prevent contrast-induced nephropathy (ATTEMPT): study design and protocol for the randomized, controlled trial, the ATTEMPT, RESCIND 1 (First study for REduction of contraSt-induCed nephropathy following carDiac catheterization) trial. Am Heart J. 2016;172:88-95.

5. Droppa $\mathrm{M}$, Desch $\mathrm{S}$, Blase $\mathrm{P}$, et al. Impact of $\mathrm{N}$ acetylcysteine on contrast-induced nephropathy defined by cystatin $\mathrm{C}$ in patients with ST-elevation myocardial infarction undergoing primary angioplasty. Clin Res Cardiol. 2011;100:1037-43.

6. Thayssen P, Lassen JF, Jensen SE, et al. Prevention of contrast-induced nephropathy with $\mathrm{N}$-acetylcysteine or sodium bicarbonate in patients with STsegment-myocardial infarction: a prospective, randomized, open-labeled trial. Circ Cardiovasc Interv. 2014;7:216-24.

7. Navarro RB, et al. The effect of spironolactone on acute kidney injury after cardiac surgery: a randomized, placebo-controlled trial. Am J Kidney Dis. 2016. https://doi.org/10.1053/j.ajkd.2016.06. 013.

8. Mejia-Vilet JM, et al. Renal ischemia-reperfusion injury is prevented by the mineralocorticoid receptor blocker spironolactone. Am J Physiol Renal Physiol. 2007;293:F78-86.

9. Sanchez-Pozos K, et al. Recovery from ischemic acute kidney injury by spironolactone administration. Nephrol Dial Transplant. 2012;27:3160-9.

10. Pretorius $\mathrm{M}$, et al. Angiotensin-converting enzyme inhibition or mineralocorticoid receptor blockade do not affect prevalence of atrial fibrillation in patients undergoing cardiac surgery. Crit Care Med. 2012;40(10):2805-12.

11. Briguori $\mathrm{C}$, et al. Cystatin $\mathrm{C}$ and contrast-induced acute kidney injury. Circulation. 2010;121:2117-22.

12. Kellum JA, Lameire N, Aspelin P, Barsoum RS, Burdmann EA, Goldstein SL, Herzog CA, Joannidis M, Kribben A, Levey AS, MacLeod AM, Mehta RL, Murray PT, Naicker S, Opal SM, Schaefer F, Schetz M, Uchino S. Kidney disease: improving global outcomes (KDIGO) acute kidney injury work group. KDIGO clinical practice guideline for acute kidney injury. Kidney Int Suppl 2012;2:1-138.

13. Tong J, et al. Neutrophil gelatinase-associated lipocalin in the prediction of contrast-induced nephropathy: a systematic review and meta-analysis. J Cardiovasc Pharmacol. 2015;66:239-45.

14. Sarwar CM, Papadimitriou L, Pitt B, et al. Hyperkalemia in heart failure. J Am Coll Cardiol. 2016;68(14):1575-89.

15. Butler J, Anstrom KJ, Feller M, et al. Efficacy and safety of spironolactone in acute heart failure: the ATHENA-HF randomized clinical trial. JAMA Cardiol. 2017. https://doi.org/10.1001/jamacardio. 2017.2198.

16. Al-Tu'ma FJ, et al. The role of serum cystatin $\mathrm{C}$ in the early detection of contrast-induced nephropathy after coronary intervention. J Kidney. 2017;3:154. https://doi.org/10.4172/2472-1220. 1000154 .

17. Nassir FS. Contrast-induced nephropathy in diabetic and non-diabetic patients after coronary intervention. J Babylon Univ/Pure Appl Sci. 2014;22(9):2530-46.

18. Taher, et al. Predicting contrast-induced nephropathy post coronary intervention: a prospective cohort study. Egypt Heart J. 2015;67:337-43. 
19. Silver SA, et al. Risk prediction models for contrastinduced nephropathy: systematic review. BMJ. 2015;351:h4395.

20. Dong Y, et al. How strong is the evidence for sodium bicarbonate to prevent contrast-induced acute kidney injury after coronary angiography and percutaneous coronary intervention? Medicine. 2016;95(7):e2715.

21. Brar SS, et al. Haemodynamic-guided fluid administration for the prevention of contrast-induced acute kidney injury: the POSEIDON randomised controlled trial. Lancet. 2014;383(9931):1814-23.
22. Butler $\mathrm{J}$, et al. Rationale and design of the aldosterone targeted neurohormonal combined with natriuresis therapy in heart failure (ATHENA-HF) trial. JACC Heart Fail. 2016;4(9):726-35.

23. Ferreira JP, Santos M, Almeida S, Marques I, Bettencourt $\mathrm{P}$, Carvalho $\mathrm{H}$. Mineralocorticoid receptor antagonism in acutely decompensated chronic heart failure. Eur J Intern Med. 2014;25:67-72.

24. McInnes CT, et al. Spironolactone dose-response relationships in healthy subjects. Br J Clin Pharm. 1982;13:513-8. 